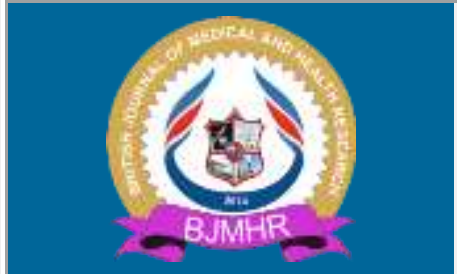

\title{
BJMHR
}

British Journal of Medical and Health Research Journal home page: www.bjmhr.com

\section{Glycerol to Prevent Corneal Haze Post Photorefractive Keratectomy Surgery in High Diopters: Biochemical and Physiological basis.}

\author{
Leopoldo Garduño-Vieyra ${ }^{1}$, Isabel De la Fuente-Batta ${ }^{2}$, Luis Antonio Rivera-Abril ${ }^{\mathbf{1}}$, \\ Luis M. Zamora-y- Cornejo ${ }^{1}$, Francisco J. Rodríguez-Ramirez ${ }^{1}$, María Teresa Zavala \\ Martínez ${ }^{3}$. \\ 1. Oftalmología Garduño, Ophthalmology Clinic. Av. Leon 428.Jardines del Moral. CP \\ 37170 Leon, Guanajuato, Mexico. \\ 2.Department of Medicine and Nutrition, University of Guanajuato, St. 20 de enero 929, \\ Obregon, CP 37320, Leon, Guanajuato. \\ 3.Clinica David, Ophthalmology Clinic. Blvd. Garcia de Leon 598, Nueva Chapultepec, \\ CP 58280, Morelia, Michoacan, Mexico.
}

\section{ABSTRACT}

The purpose of this study is to find an effective treatment without corneal haze in patients with high diopters and/or thin corneas; the idea is to offer them a safe surgical refractive treatment to improve their visual acuity (VA) without the use of intraocular implants for higher diopters avoiding therefore any associated risk. A retrospective, observational and descriptive case series included 75 eyes with ametropias with spherical equivalents the lowest -5.00 Diopters, the highest -22.00 Diopters who underwent photorefractive keratectomy. They were treated additionally with mitomycin $0.02 \%$, contact lens, tobramycin/dexamethasone, and sodium hyaluronate for seven days. Upon removal of the contact lens, they were treated with glycerol $0.9 \%$ and sodium hyaluronate three times a day for two months. The 75 eyes included had a preoperative visual capacity was of $1.57 \log$ MAR (SD 0.5), and postoperative of 0.1 logMAR (SD .12) (T test for paired samples, $\mathrm{p}=000$ ) (FIGURE 2). Preoperative pachymetry was of 564.7 (SD 43.3) and postoperative of 449.1 micra (SD 55.9), ( $\mathrm{T}$ test for paired samples, $\mathrm{p}=0.000$ ). There were 5 cases of haze at the first postoperative month, no case of haze was presented at 3,6 and 12 months of follow-up. This study shows that the use of glycerol is an effective treatment as an adjuvant to prevent haze; based on its osmolarity, hygroscopic capacity and its protein stabilizing properties.

Keywords: Glycerol. Hygroscopic. Haze. Keratocytes. Ametropia.

Please cite this article as: Vieyra LG et al., Glycerol to Prevent Corneal Haze Post Photorefractive Keratectomy Surgery in High Diopters: Biochemical and Physiological basis . British Journal of Medical and Health Research 2019. 


\section{INTRODUCTION}

Photorefractive Keratectomy(PRK) is one of the most common techniques to correct myopia, hypermetropia and astigmatism. This procedure consists in the remission of the epithelium with a $20 \%$ alcohol dilution, for a maximum of 35 seconds, followed by the ablation in the most anterior part of the cornea with the Excimer Laser (1).

The formation of an adjacent hypercelullar fibrotic stromal scar on the epithelium surface (haze) may be found; it would be seen as a grayish area of sub-epithelial tissue.

In PRK we often find that as more tissue is consumed, the larger the pattern of subepithelial central corneal haze is $(2,3,4,5,6$, and 7$)$.

\section{MATERIALS AND METHOD}

This retrospective, observational, and descriptive series of cases was carried out from January 2016 to March 2018, at the Clínica de Oftalmología Garduño, Department for Corneal Services, in Irapuato-León, Mexico. The study included 75 eyes with high ametropias with a spherical equivalent from, the lowest $-5.00 \mathrm{D}$ to the largest $-22.00 \mathrm{D}$. Most of these patients came to this Corneal Services Department because they had been rejected as candidates for refractive surgery in other places. Most of these patients were denied Lasik and/or PRK surgery because of high ametropias and the width of their cornea, or they had been proposed to have refractive surgery with anterior camera lens for high myopia. Directed clinical history was gathered and they were specifically asked if they were contact lenses users, if they had already undergone a refractive treatment, and the reason why surgery had been denied to them. Their evaluation included preoperative visual acuity, refraction, biomicroscopy, intraocular tension, eye bottom revision, pachymetry, topography, and keratometry. Initially topical anesthesia was applied 3 minutes before surgery on the cornea (tetracaine drops $0.1 \%$ ); followed by ethylic alcohol at $20 \%$ for 20 seconds in a $9.00 \mathrm{~mm}$ Thornton ring to remove the corneal epithelium with merocel sponges, after drying the cornea the ablation of the tissue was done with scan laser Nidek EC5000 (Japan, Nidek Inc. 47651 Westinghouse Drive, Freemont, CA 94539). The ablation area was cleaned with mitomycin at $0.02 \%$ for 15 seconds and posteriorly washed with balanced saline solution for five seconds. At the end of the procedure a therapeutic contact lens was used to cover the cornea. The postsurgical management was made with a colirio of tobramycin 3mg/dexamethasone $1 \mathrm{mg}$ per $\mathrm{ml}$ is used three times per day, for ten days; sodium hyaluronidate $4.0 \mathrm{mg} / \mathrm{ml}$ every 2 hours for one month, sodium ketorolac $5.0 \mathrm{mg} / \mathrm{ml}$ three times per day for 4 days; after 7 days the therapeutic contact lens is removed and the patient starts using glycerol with sodium hyaluronidated (Zolag: glycerin 0.9\%/ sodium hyaluronidated 0.4\%, Lab. Grin A Lupin Group Company; Rodríguez Saro \#630 col del Valle. Mex. D.F.) three times per day, during two months, and then sodium hyaluronidate of $4.0 \mathrm{mg} / \mathrm{ml}$ every 4 
hours for one more month. All procedures used in the subjects of this study were done after having informed consent.

\section{RESULTS AND DISCUSSION}

75 eyes were included. Average refractive values are presented in TABLE 1 . The preoperative visual capacity was of $1.57 \log$ MAR (SD 0.5), and postoperative of $0.1 \log$ MAR (SD .12) (T test for paired samples, $\mathrm{p}=000$ ) (FIGURE 1). Preoperative pachymetry was of 564.7 (SD 43.3) and postoperative of 449.1 micra (SD 55.9), (T test for paired samples, p=0.000) (FIGURE 2). 5 cases presented haze one month after surgery; there were no haze cases after 3, 6 and 12 months of follow up.

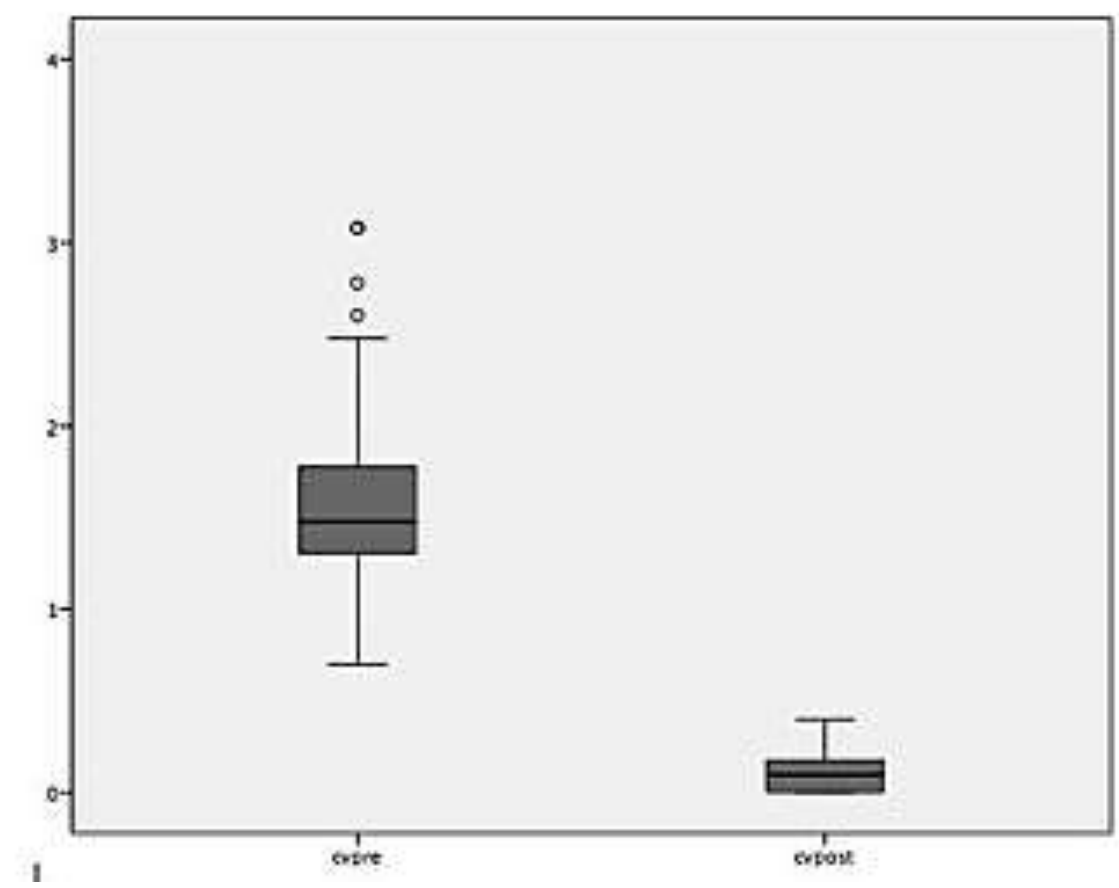

Figure 1: Box plot showing the visual capacity distributions pre and postoperative 


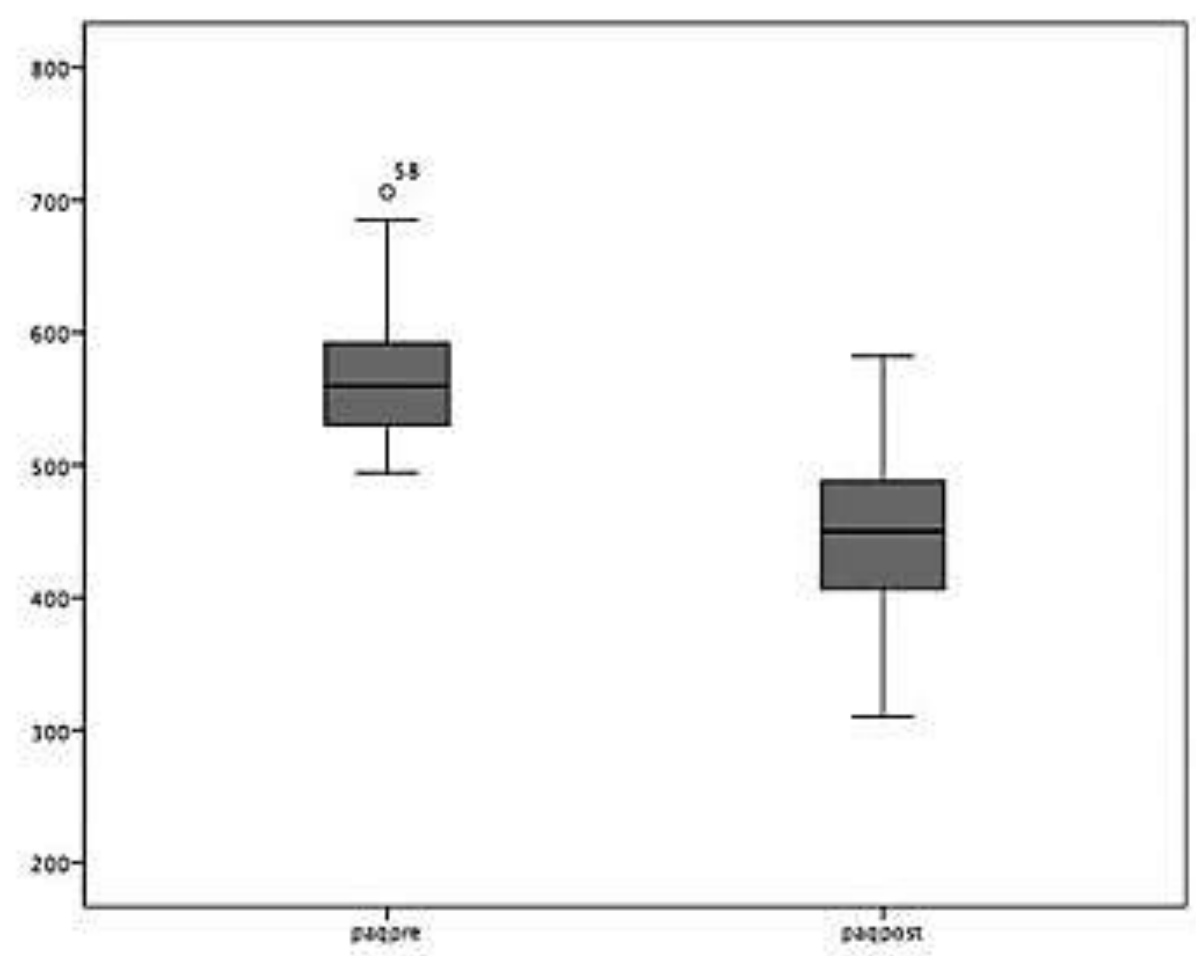

Figure 2: Box plot showing the distributions of the pre and postoperative pachymetries Table 1: Pre-operative refractive values

\begin{tabular}{llllll}
\hline & N & Minimum & Maximum & Mean & SD \\
\hline Sphere & 74 & -21.00 & -2.00 & -7.75 & 3.68 \\
Cylinder & 66 & -6.00 & -0.50 & -2.44 & 1.49 \\
\hline
\end{tabular}

Since 1957, Maurice suggested the Lattice Theory to explain the transparency of the cornea and demonstrated that the collagen fibers are arranged in a sort of lattice; with inter fibrils spaces smaller than the light wavelength. The above is valid in the maintenance of the equidistant arrangement of the different stromal lamellae (1)(8).

The human cornea is the one that imbides less fluid, however it absorbs an equivalent to 2.5 its own weight; the consistence of a totally embedded cornea is similar to the vitreous body and stops being transparent, the swelling is a result of the large amount of water distributed throughout the whole stromal surface and perpendicularly to its surface $(1,8)$.

Haze (opacity, nebula, mist) is a complication of PRK resulting from the superficial reticulated scarring of the stroma immediately subjacent to the epithelium covering the ablation zone; reticulated opacity is a normal part of the repairing biological corneal process and some of the most relevant pathological aspects are absence of the Bowman's membrane, type 3 collagen deposit, absence of keratan-sulfate in the extracellular matrix; disorder in the spatial distribution of collagen fibers, large amounts of fibronectin and laminin, increase in number and size of keratocyte in the area of treatment. Corneal haze reaches its peak three months after surgery with a tendency to continue gradually reducing over one year and a half after surgery. 
PRK is one of the most common techniques to correct myopia, hypermetropia and astigmatism. This procedure consists in the remission of the epithelium with a $20 \%$ alcohol dilution, for a maximum of 35 seconds, followed by the ablation in the most anterior part of the cornea with the Excimer Laser (1). There are reports of reepithelization alterations by transmission electronic microscopy and the TUNEL staining as a result of ultra structural changes in the basal and Bowman membranes, modifications in cellular proliferation and adherence of the basal layer of the epithelium $(2,3)$. In PRK there is focal hypertrophy of the basal cells with epithelial hyperplasia and always in the zone treated with Excimer and with some thick stromal scarring; there are changes in the elongation and hypertrophy of the cells in the basal layer of the epithelium, or hyperplasia of the epithelium in the corneal surface; this was registered with thickness measurements with confocal microscope. The more tissue used, the more we find a central-corneal subepithelial haze pattern $(2,3,4,5,6,7)$. With confocal microscopy and histopathological evaluations immediately after surgery and 6 months later, we have found epithelial and stromal damage followed by apoptosis of the

keratocytes around the lesion; later there is transitory infiltration of acute and chronic inflammatory cells, proliferation and migration of epithelial cells and surviving keratocytes, reformation of union complexes, differentiation of fibroblasts to myofibroblasts for synthesis, cleaning and contraction of the wound originating the haze $(3,6,7,9)$.

Keratocytes develop from the neural crest of the cells from which they migrate to install themselves in the mesenchyme; once the keratocyte is placed in the stroma it starts to synthesize the different types of collagen molecules (I, V,VI) and keratan sulfate, which are usually found in the cornea at rest. According to a study, the median density of the keratocyte in the human stroma is approximately 20,500 cells per cubic millimeter, or 9,600 in one column of one square millimeter in the section. The highest density is in the $10 \%$ higher of the stroma and it goes down in number with the age, about $0.45 \%$ per year $(2,3,4)$.

The expansion of the corneal stroma by groups of mucopolysaccharides may be compensated or neutralized with opposite charge ions; these will create an osmotic effect which will provoke the absorption of water, originating therefore the corneal stroma retraction. Likewise, for the case of negative charges the mucopolysaccharides would also cause the repulsion force amongst the different molecules that tend to separate each other, provoking the absorption of the fluid (8). This fluid absorption will favor the increase in the synthesis of the fibers in the extracellular matrix by the action of the fibroblasts, which are cells residing in the connective tissue, the precursors of the keratocyte, mesenchymal pluripotent cells that can transform into fibroblasts. After a corneal lesion some of the keratocytes will present apoptosis, motivated by the signaling molecules secreted by the superior layers, such as interleukin 1 alpha (IL1 alpha) 
and Tumor Necrosis Factor alpha (TNF-alpha). Other neighboring active keratocytes proliferate and start synthesizing metalloproteinases from the matrix causing tissue remodeling. These activated cells are pointed out as keratocyte or fibroblast activity or, as it is also expressed, it assumes a repairing phenotype. In heavier or advance-stage lesions, the healing process is transformed. The myofibroblasts actively secrete the extracellular matrix (ECM); this transformation is believed may be caused by the Beta transforming Growth Factor (TGF-beta). As soon as the basal membrane of the corneal epithelium is restored, the TGF-beta drastically reduces the affluence in the stroma and the fibroblasts disappear, then the remaining activated keratocyte continue for a while remodeling the ECM, secreting IL1-alpha with the purpose of maintaining the repairing phenotype (5).

Glycerol has been used for years by biochemists to stabilize the activity of the enzymes and the structure of proteins (10). The stability of proteins in aqueous solution is potentiated routinely by means of co-dissolvers like glycerol. Glycerol inhibits protein aggregation (acid mucopolysaccharides) during the refolding of many of them (11). Their function is limited as result. Glycerol is one of the substances better tolerated by the cornea, even for its preservation $(12,13)$. Keratocytes inhibit their conversion into fibroblasts by the action of the glycerol or glycerine I, as a result of their molecular composition made of C3H8O3 (Greek: Glycos=sweet) also known as propanetriol is one of the main products of digestive degradation in lipids.

This article reports the characteristics of the patients operated with PRK and their incidence of clouding or haze, as well as the postoperative management to avoid opacification of the cornea using intraoperative mitomycin and glycerine in the postoperative for two months.

Haze incidence varies according to different authors and severity, presenting the highest peaks of incidence between the first and sixth month after PRK surgery, this being time dependent, and the tendency exists between 12 and 18 months $(3,5,14,15)$. The anterior stroma starts regenerating days after PRK, when the activated keratocytes migrate to the ablated stromal bed during the first three weeks. These activated keratocytes transform into fibroblasts and are associated with an increase in the extracellular matrix as they are the ones responsible for the formation of new collagen. In general, it is believed that surgically-induced apoptosis, keratinocyte proliferation, the activation and posterior transformation in fibroblasts are phenomena that regulate the scarring process and the appearance of haze $(16,17)$. In the presence of inappropriate scarring, large amounts of activated keratocyte, and a large production of extracellular matrix, we have what we usually call haze, creating the complication seen as corneal transparency reduction and visual quality diminished $(16,18,19$, 9). Haze or corneal clouding appears after Photorefractive Keratectomy in $2-4 \%$ of the eyes, depending on the level of correction, even though most patients have a transitory haze (20). 
Herch et al. after two years of follow up of 701 eyes with PRK, found a light haze in $22.5 \%$, a medium haze in $3.3 \%$, a moderate haze in $1.5 \%$ and only $0.5 \%$ presented severe haze. Loewensteinv et.al. detected haze in $3 \%$ of the 825 patients with PRK for myopia, after twelve months of follow up $(17,21)$. Kaiserman et al. studied factors associated with the development of haze after a PRK and found a significant correlation with hypermetropia, high myopia and high astigmatism. In a total of 7335 eyes of 3854 patients with PRK, during a two year period, using the epithelium removal technique with alcohol or laser, applying mitomycin for 20-60 seconds. Highest haze incidence was found in patients with hypermetropia (10.8\%), eyes with myopia with more than 6 diopters had double incidence of haze (2.1\%) compared against eyes with low and moderate myopia $(1.1 \%)$, likewise, patients with preoperative astigmatism of more than 3 diopters had more incidence of corneal clouding than those with low astigmatism $(2.9$ Vs $1 \%)(22,23,24,18)$. The epithelium removal method also plays an important role: haze incidence was 2.6 times higher with the use of alcohol compared against laser. Authors mentioned that it may have to do with the toxic effect of alcohol on epithelial cells and the anterior stroma, including the edges of the wound (24). Post PRK corneal clouding is a serious condition for the patient; it could cause permanent and severe visual loss (3). Secondary haze after PRK is characterized by superficial stromal opacity and, histologically, by a subepithelial fibrosis $(3,19,25)$.

Possible factors associated such as the amount of resected tissue, laser type, medications, gender, race, iris color and allergies are some mechanisms that could participate in the production of corneal clouding $(3,22,23,25)$.

Mitomycin $\mathrm{C}$ has an anti-proliferative effect resulting from the capacity to generate covalent unions in the DNA chains and, on the other side, a cytotoxic effect which unlocks an increase of keratocyte apoptosis when applied on the cornea. For the case of surface surgery, as is the case of this work, it is used to reduce incidence and intensity of postoperative clouding and fibroblastic proliferation in the corneal stroma (18). Many authors have shown that, after a PRK, the epithelium not only regenerates but it also suffers postoperative hyperplasia, which is considered the most probable cause of enlargement of the corneal width detected after surface ablation. However, there are other authors who did not detect that epithelial hyperplasia, but a stromal thickening which justifies the postoperative increase in the cornea width (26). It has also been shown that mitomycin $\mathrm{C}$ does not seem to annul the corneal thickening after the surface ablation; this is what Benito et al. have shown (27).

In this work glycerine in ophthalmic drops was used in postoperative as aid to inhibit the presence of haze, because it has a hyperosmotic effect. Nutrient metabolic exchange in the cornea is slowed down with glycerol because of its molecular weight and its osmolarity, the 
above optimizes both; glycerol itself gives glucose to the fibroblasts, and as they are totipotential they will turn into keratocytes and will provide transparency to the cornea. Glycerol (C3 H3 O3) is a polyalcohol, commercially known as glycerine, used in many products as emollient, humectant, dissolvent and lubricant. The moisture regulating effects of glycerin work in opposite directions. Glycerin, for example, may act as a humectant in certain foods and this is done by acting as a hygroscopic agent, which means that it obtains the moisture from the environment. However, under certain conditions it behaves as a hyperosmotic agent, avoiding protein aggregation, inhibiting the generation of the subepithelial fibrosis and as a consequence absence of corneal clouding; as shown in the 12-month follow-up results of the 75 eyes operated with the Photorefractive Keratectomy with ametropias with an spherical equivalence of, the lowest 5 diopters and, 22 the highest $(10,12,13,28,29,30)$.

Limitations of the study: for this study on a series of retrospective cases we did not have a control group, so the level of evidence is reduced and the following questions arise:

Which concentrations are ideal? How many times per day and for how long are the ideal postoperative recommendations for this treatment?

\section{CONCLUSION}

In order to correct refractive errors many surgeons decide to remodel the cornea with PRK, either because of thin pachymetry, doubtful topographies or the high certainty to obtain appropriate corneal width. Haze appearance between early postoperative and a year later is expected after PRK. Currently, surgeons must carefully assess pre, intra and post operative parameters to reduce to a minimal all possibilities of complication as PKR is done on healthy eyes. Keratometry, pachymetry, topography and real value measurements of ametropia are fundamental for the depth of the ablation; more ablation is needed for more refractive error, but this increases the possibilities of haze; this study was performed on the results of PRK with high ametropias. This work shows that the use of propanetriol (glycerine or glycerol) combined with sodium hyaluronate colirio, three times a day, for two months after PRK surgery is an efficient treatment as aid to avoid haze; based upon its hygroscopic capacity, its osmolarity, the effects on the corneal stroma, the stabilization of proteins present in the lesion and growth factors. More random controlled clinical studies are needed in order to see the actual impact of glycerin in PRK patients with high refractive errors.

\section{REFERENCES}

1. Duke-Elder, Sir Stewart. The problem of corneal transparency. In The transparency of the cornea. 1960. Duke-Elder, Sir Stewart, and Perkins E.S. (eds.. 1958: 1

2. Harris, J. E.and Nordquist, L. T. The hydration of the cornea. I. the transport of water from the cornea. Amer. J. Ophthal. 1955; 40, (2), 100. 
3. Lee JB, Javier JA, Chang JH ChenCC, Kato T, Azar DT. Confocal and electron microscopic studies of laser subepitelial keratomileusis (LASEK) in white leg horn chick eye. Arch Ophthalmol. 2002; 120, 1700-06.

4. Guerra Arturo, Velasco Regina, Baca Oscar. Comportamiento epitelial corneal post PRK evaluado con microscopio confocal. Rev. Mex Oftalmol; January-February 2008;82(1):9-12.

5. Vesaluoma M, Pérez-Santola J, Petroll WM y cols. Corneal stromal changes induced by miopic LASIK. InvestOphthalmol Vis Sci. 2000; 41:369-376.

6. Vincent Vageneda, Miranda; GS Yap, Ben Hardt L. Trout. Biochemistry. Mecanismo de estabilización de proteinas y prevención de la agregación de proteínas por glicerol. ACSPub. 2009; 48(46), 11084-11096.

7. KunihikoGekko and SergeN.Timasheff. MechanismofProteinStabilizatiónby Glicerol: Preferential Hydration in Glycerol-Water Mixtures. Biochemistry. 1981; 20, 46674676.

8. Wilson SE, SS Chaurasia, Medeiros FW. Apoptosis en la iniciación, la modulación y la terminación de la respuesta de la cicatrización corneal. Exp. Ojo. Res. 2007; 85(3):305-11.

9. West-Mays JA, DwivediDJ. The keratocyte: corneal stromal cell with variable repair phenotypes. Int J. Biochem. Cell Biol. 2006; 38(10):1625-31.

10. Patel S, McLaren J,D Hodge, Bourne W. Normal human keratocyte density and corneal thickness measurement by using confocal microscopy in vivo. Inverir. Ophthalmol. Vis. Sci. Feb 2001; 42(2): 333-9.

11. Boume WM, Shearer DR, Nelson LR. Human corneal endotelial tolerancetoglycerol, dimethylsulfoxide, 1,2-propanediol, and2,3-butanediol. Cryobiology. Feb 1994;31(1):1-9.

12. Rich SJ, Armitaje WJ. Corneal tolerance of vitrifiable concentrations of glycerol. Cryobiology. Apr 1992; 29(2):153-64.

13. Erie JC, Patek SV, Mc Laren JW, Maguirre LJ y cols. Keratocyte density in vivo after photorefractive keratectomy in humans. Trans Am Ophthalmol Soc. 1999; 97: 221-36.

14. Rojas E., González, J: Microscopia confocal cuantitativa del haze corneal y correlación con la ametropía a tratar en cirugía refractiva de superficie. Revista Mexicana de Oftalmología, Jul- Aug 2016; 90(4): 153-208.

15. Z. Shalchi, D.P. O Brant, R.J. Mc.Donald et al. Eighteen years follow up of excimer Laserphotorefractive. J. Cataract Refract Surg. 2015; 41: 23-32. 
16. O.M. Tirado, A. Hernández. Uso de la mitomicina en la prevención del haze corneal. Rev. Cubana Oftalmol. 2011; 24, 1, 100-110.

17. C. Winklen, U. Reischi, C. Lohmann. Corneal haze after photorefractive keratectomy

1. for myopia. Role of collagen IV MRNA typing as predictor of haze. J. Cataract Refract Surg. 2002; 28, 1446-1451.

18. U. Celik, E Bozkurt, B.Celik et al. Pain. Wound healing and refractive comparison of mechanical and transepithelial debridement in photo refractive keratectomy for myopia: results in 1 year follow up. Contact lens and anterior eye. 2014; 37, 6, 420426.

19. J.L. Aliu, F. Soria, A. Abbouda, et al Laser in situ Keratomileusis for 6.00 to 18 Diopters of myopia and up to 5.00 diopters of astigmatism: 15 years follow up. $J$. Cataract Refract surg. 2014; 41, 33-40.

20. M. Bohnke, A. Thaer, I. Schipper Confocal Microscopy reveals persisting stromal Changes after myopic photorefractive Keratectomy in zero haze corneal. $\mathrm{Br} . \mathrm{J}$ Ophthalmol. 1998; 82, 1393-1400

21. M. Mimouni, I. Vainer, Y. Shapira, et al. Factors Predicting the Need for Retreatment After Laser Refractive Surgery. Cornea. 2016; 35, 5, 607-612.

22. Carones F. Scandols E, Vacchinil. Evolution of the prophylactic use of mitomycin C to Inhibit haze formation after photorefractive keratectomy. J. Cataract Refract surg. 2002: 28, 2088-95.

23. Gipson IK. Corneal epitelial and stromal reactions to excimer laser photorefractive Keratectomy. I concerns recording the response to the corneal epithelium to Excimer laser ablation. Arch Ophthalmol. 1990; 108 1539-1540.

24. Dawson DG, Edelhauser HF, grossaklausHE. Long term Histopathologic findings in human corneal wounds after refractive surgical procedures. AM J. Ophtlamol. 2005; $139,168-178$.

25. Lohman CP. Reisch U. Marshall J. Regression and epithelial hyperplasia after myopic Photorefractive Keratectomy in human cornea. J: Cataract refract surg. 1999; 25, $712-5$.

26. De Benito L. Teus MA, Drake T. Effect of mitomycin C on corneal regrowth after laserassisted sub-epithelial keratectomy (LASEK). Arch Soc. Esp. Oftalmol. 2011; 86(7): 213-7.

27. Kong, P.S. Wan Daud, W. M.A Conversion Of crude and pure glycerol in to Derivates a feasibility evoluation. Review. Sust.Energ. Rev. 2016; 63: 533-555. 
28. Vanegende V tap MG trout BL. Mechanisms of protein stabilization and prevention of protein agregation by glycerol. Biochemistry. 2009 Nov 24; 48 (46) 11084-96.

29. Netto, MV: Mohan Wilson. Stromal haze, miofibroblasts and surfact irregularity after PRK. Exp Eyes Res. 2006 May; 82(5): 788-797.

30. Herah PD, Stulfing RD; Steinert Rf. Waring go III Thompson KP. O Connell M. Doney; K, Schein LD. The Summit PRK study group results of phase III Excimer Laser photorefractive Keratectomy for myopia. Ophthalmology. 1997; 104: 1535-1553.

\section{BJMHR is \\ - Peer reviewed \\ - Monthly \\ - Rapid publication \\ - Submit your next manuscript at editor@bjmhr.com

